\section{Biosynthesis of Armentomycin: A Chlorinated Nonprotein Amino Acid}

Sir:

Although over 1,500 halogenated natural products have been identified in animals, plants, and microorganisms, ${ }^{1,2)}$ only two biological routes for their formation are known: the direct methylation of halide ions, ${ }^{3}$ ) and haloperoxidase-catalyzed halogenation. ${ }^{2,3}$ The biosynthesis of armentomycin (L-2-amino-4,4-dichlorobutanoic acid, 6), a nonprotein amino acid with antibiotic properties $^{4)}$ is of particular interest because no mechanism is known for the chloroperoxidase-catalyzed insertion of chlorine substituents remote from other functional groups. ${ }^{2)}$ We present here the initial results from our investigation of armentomycin biosynthesis in Streptomyces armentosus var. armentosus.

A starch, L-lysine and $\mathrm{KCl}$ medium $(100 \mathrm{ml} / 500-\mathrm{ml}$ Erlenmeyer flask) was inoculated with a 48-hour seed culture of S. armentosus var. armentosus UC2862 and incubated at $27^{\circ} \mathrm{C}$ with rotary shaking $(220 \mathrm{rpm}){ }^{5)}$ ${ }^{13} \mathrm{C}$-Labelled substrates $(1.8 \sim 2.7 \mathrm{mmol})$ were added in four equal portions at ca. 48, 72, 96, and 120 hours, and the cultures were harvested 24 hours after the last addition. Armentomycin was isolated by cation-exchange chromatography (Amberlite IR-120, $\mathrm{H}^{+}$form, $0.3 \mathrm{M}$ pyridine elution) and derivatized with $p$-toluenesulfonyl chloride and ethereal diazomethane. $N$-Methyl- $N$ tosylarmentomycin methyl ester was purified by preparative-layer chromatography (silica gel, developed sequentially in hexane - EtOAc, $80: 20 \mathrm{v} / \mathrm{v}$, and benzene $\mathrm{MeOH}, 99: 1 \mathrm{v} / \mathrm{v})$. Isotopic enrichments were measured by ${ }^{13} \mathrm{C}$ NMR spectroscopy (62.9 MHz). The extent of isotopic labelling at each carbon atom, expressed as $\%{ }^{13} \mathrm{C}$ enrichment above the $1.1 \%$ natural abundance of this isotope, was calculated from ${ }^{13} \mathrm{C}$ NMR peak heights normalized to the natural abundance signals provided by the derivatization reagents. ${ }^{6)}$ The standard deviation of these measurements is $\leq 0.2$.

$N$-Methyl- $N$-tosylarmentomycin methyl ester from cultures fed $\left[1-{ }^{13} \mathrm{C}\right]$ acetate was labelled efficiently and exclusively at C-1 (15\% enrichment); cultures fed $\left[2-{ }^{13} \mathrm{C}\right]$ acetate gave armentomycin highly enriched at C-2 $(14 \%)$. Enriched $(7 \%)$, coupled $\left({ }^{1} J_{\mathrm{CC}}=61 \mathrm{~Hz}\right)$ signals for C-1 and C-2 of $N$-methyl- $N$-tosylarmentomycin methyl ester demonstrated the intact incorporation of $\left[1,2-{ }^{13} \mathrm{C}_{2}\right]$ acetate. In the cultures fed $\left[2-{ }^{13} \mathrm{C}\right]$ - or $\left[1,2-{ }^{13} \mathrm{C}_{2}\right]$ acetate, smaller but equal enrichments $(<4 \%)$ at C-3 and C-4 of armentomycin indicated that C-2 of acetate was incorporated into this half of the molecule via intermediates of the citric acid cycle. ${ }^{6)}$ Since the methylene carbon of oxaloacetate is a possible site for chloroperoxidase-catalyzed chlorination, ${ }^{2)}$ labelled oxaloacetate was supplied by feeding DL- $\left[3-{ }^{13} \mathrm{C}\right]$ aspartic acid. The small and approximately equal enrichment $(1 \sim 1.5 \%)$ of all carbon atoms in the armentomycin obtained indicated that oxaloacetate was not a direct precursor. The pattern of incorporation suggested equilibration via a symmetrical intermediate of the citric acid cycle; both C-1, C-2 and C-3, C-4 of armentomycin could then be derived from phosphoenolpyruvate (1) or pyruvate (2) formed from oxaloacetate. Acetyl-CoA (4) derived from oxaloacetate labelled at C-2 and C-3 would be equally labelled at both carbons, and would account for enrichment of $\mathrm{C}-1$ and $\mathrm{C}-2$ of armentomycin. Concurrent incorporation of the $\mathrm{C}-2, \mathrm{C}-3$ labelled phosphoenolpyruvate or pyruvate into $\mathrm{C}-3$ and $\mathrm{C}-4$ of armentomycin would explain the similar enrichment of all four carbons.

The postulated precursor role of phosphoenolpyruvate and pyruvate was tested by feeding $\left[2-{ }^{13} \mathrm{C}\right]$ glycerol and $\left[2-{ }^{13} \mathrm{C}\right]$ pyruvate. The similar enrichments (8 and 7\%) at $\mathrm{C}-1$ and $\mathrm{C}-3$ of armentomycin derived from glycerol confirmed the hypothesis, and supported the idea that C-1, C-2 and C-3, C-4 of armentomycin are derived from a common precursor. In the $\left[2-{ }^{13} \mathrm{C}\right]$ pyruvate experiment, ${ }^{13} \mathrm{C}$ enrichments at both $\mathrm{C}-1 \quad(5 \%)$ and $\mathrm{C}-3(4 \%)$ of armentomycin indicated that pyruvate rather than phosphoenolpyruvate was the last intermediate common to the two $\mathrm{C}_{2}$ units of armentomycin.

In the biosynthetic pathway proposed (Fig. 1), pyruvate (2) is converted to acetyl-CoA (4) and a chlorinated intermediate, such as dichloropyruvate (3); subsequent condensation of these two units provides an intermediate (5) that can be converted to armentomycin (6) by dehydration/hydration, oxidation/decarboxylation, and transamination reactions analogous to those in the chain-extension sequences of the biosynthetic

Fig. 1. Hypothesis for the biosynthesis of armentomycin (6) from pyruvate (2).

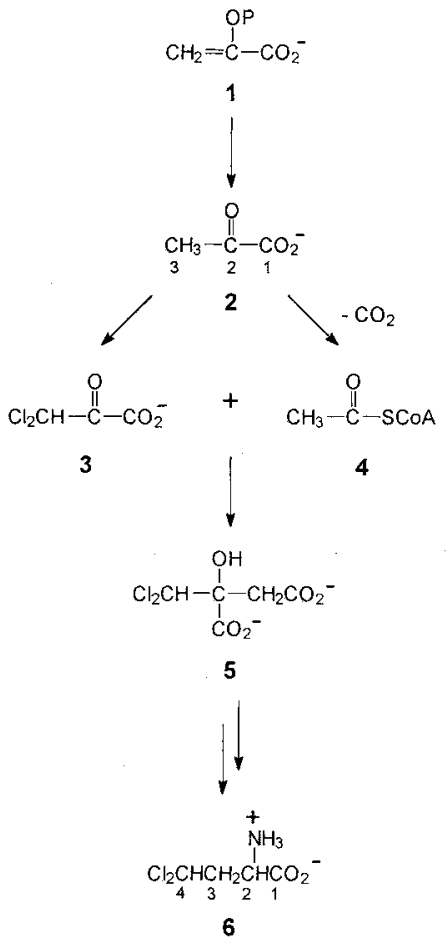


pathways to lysine and leucine. ${ }^{7)}$ Armentomycin is a structural analogue of leucine, and dichloropyruvate is the corresponding analogue of the leucine precursor, $\alpha$-ketoisovalerate. However, the dehydration/hydration and oxidation/decarboxylation sequence is similar to that catalyzed by citric acid cycle enzymes in the pathway to phosphinothricin, the nonprotein amino acid residue in the herbicidal tripeptide bialaphos. ${ }^{8)}$ Whether enzymes in the citric acid cycle or the leucine pathway, or a distinct set of secondary metabolism enzymes, are used for armentomycin biosynthesis must now be addressed.

\section{Acknowledgments}

Financial support was provided by research grants (to L. C. V. and R. L. W.) from the Natural Sciences and Engineering Research Council of Canada (NSERC), and by an exchange fellowship (to J.-Y. H.) from the World University Services of Canada. We are indebted to the Atlantic Region Magnetic Resonance Centre for providing NMR spectra.

\section{KAOQIN LIU ROBERT L. WHITE* JIAN-YONG $\mathrm{HE}^{\dagger}$ LEO C. VINING ${ }^{\dagger}$}

Department of Chemistry, Dalhousie University, Halifax, NS, B3H 4J3, Canada

+Department of Biology, Dalhousie University, Halifax, NS, B3H 4J1, Canada

(Received December 5, 1994)

\section{References}

1) GRIBBLE, G. W.: Naturally occurring organohalogen compounds--a survey. J. Nat. Prod. 55: 1353 1395, 1992

2) Neidleman, S. L. \& J. GeigerT: Biohalogenation: Principles, basic roles and applications, pp. $13 \sim 84$, Ellis Horwood, Chichester, 1986

3) HARPER, D. B.: Biogenesis and metabolic role of halomethanes in fungi and plants. In Metal Ions in Biological Systems, Vol. 29. Ed., H. Sigel \& A. Sigel, pp. $345 \sim 388$, Marcel Dekker, New York, 1993

4) Argoudelis, A. D.; R. R. Herr, D. J. Mason, T. R. PYKe \& J. F. ZieSERL: New amino acids from Streptomyces. Biochemistry 6: 165 170, 1967

5) He, J.-Y.; L. C. Vining, R. L. White, K. L. Horton \& J. L. Doull: Nutrient effects on growth and armentomycin production in cultures of Streptomyces armentosus. Can. J. Microbiol., accepted for publication.

6) White, R. L.; K. C. SMith \& A. C. DeMarco: Biosynthesis of 5-hydroxy-4-oxo-L-norvaline in Streptomyces akiyoshiensis. Can. J. Chem. 72: 1645 1655, 1994

7) Umbarger, H. E.: Amino acid biosynthesis and its regulation. Ann. Rev. Biochem. 47: 533 606, 1978

8) Shimotohno, K.; H. Seto, N. Ōtake, S. Imai \& A. Satoh: Studies on the biosynthesis of bialaphos (SF-1293) 7. The absolute configuration of 2-phosphinomethylmalic acid, a biosynthetic intermediate of bialaphos. J. Antibiotics 39: $1356 \sim 1359,1986$ 